\title{
High-Temperature Carbon-Irradiation Issues for the Sombrero ICF Reactor
}

September 15, 1999

\author{
Author: $\quad$ Tobin Munsat \\ Princeton Plasma Physics Laboratory \\ PO Box 451 \\ Princeton, NJ 08543 \\ Phone (609) 243-2941 \\ Fax (609) 243-2418
}




\begin{abstract}
In order to assess the feasibility of carbon materials for the first-wall of the Sombrero $\mathrm{KrF}$ laser-driven ICF fusion reactor ${ }^{(1)}$, published experimental results relating to mechanical and thermal properties of graphites and carbon-fiber-composites (CFC's) under neutron irradiation and high heat loads are reviewed. Results are compared to published design requirements for the Sombrero ICF reactor, with particular attention to three separate issues of concern: 1. Erosion rates of the first wall are highly sensitive to the thermal conductivity value, which is itself environment-sensitive (radiation and high temperature). Erosion rates at the first wall are calculated using a high-temperature post-irradiation conductivity value of $50 \mathrm{~W} / \mathrm{m} \cdot \mathrm{K}$, with complete erosion of the first wall layer predicted within 14 months Sombrero full-power operation (f.p.o.), illustrating the sensitivity of erosion rates to thermal conductivity assumptions. 2. Radiation-induced swelling in 2-D and 'pseudo 3-D' CFC's is consistently $\sim 20 \%$ under hightemperature neutron damage of 5 dpa (4 months f.p.o.). This level of swelling would pose technical challenges to the engineering of the target chamber modules. 3. Total tritium retention is predicted to be $\sim 0.5$ to $5 \mathrm{~kg}$ in the Sombrero chamber within 8 months f.p.o., which may call into question safety-status assumptions of the CFC-based chamber design. These results indicate the urgency of high-temperature neutron-irradiation tests of fully symmetric 3-D CFC's in order to support the plausibility of a carbon first-wall IFE chamber such as proposed for Sombrero.
\end{abstract}




\section{Background}

The effect of neutron irradiation on carbon-based materials has been reviewed elsewhere $^{(2,3,4)}$. In graphite, irradiation from fast neutrons causes displacement of carbon atoms from the lattice sites into interstitial locations, which leaves vacancies in the basal plane of the graphite. As the temperature is increased, the mobility of the interstitial carbon atoms increases, and eventually these atoms can join to form clusters or new planes. Conversely, the vacancies also become mobile at high temperatures, and can cluster and collapse.

Radiation induced damage has been shown to cause significant changes in thermal conductivity, bulk material dimensions, and retention of hydrogen isotopes, as well as other material properties. Thermal conductivity is a function of crystalline symmetry, which directly affects the mobility of phonons within the material. Elevated temperatures cause lattice vibrations and phonon scattering that lower the thermal conductivity. Radiation damage to the lattice degrades thermal conductivity by introducing defects in the lattice that act as phonon scattering centers, effectively reducing the phonon mean free path. As with dimensional changes, this effect is enhanced at higher temperatures.

Studies of irradiation of natural graphite flakes and highly oriented pyrolytic graphite have shown that there is net shrinkage in the basal plane direction coupled with growth in the direction perpendicular to the basal plane (c-axis). There is no observed limit on such dimensional changes up to very high fluence (60 dpa, see ref. 4 p. 420). This fundamental process takes place in any graphitic material including polycrystalline and carbon-fiber composite materials.

The exact nature of radiation-induced dimensional change in anisotropic graphites depends on the specific material. An example that illustrates these effects is the fine-grained 
graphite Graphnol N3 $\mathrm{M}^{(5)}$. This material has significant pore orientation in one preferred direction. The lack of the crystalline symmetry allows for the pores in one direction to accommodate the expansion of the graphite crystals upon neutron irradiation. With increasing neutron fluence, the native porosity is filled and subsequent damage induces new porosity oriented parallel to the basal planes. At this point the structure's ability to absorb expansion is 'saturated', and the material begins to re-expand. The expansion rate increases with increasing neutron fluence, and the volume expands beyond the initial (pre-irradiation) value. Cracks and pores caused by differential crystalline strain eventually cause total disintegration of the material $^{(2)}$. The optimization of N3M to resist neutron damage (because of the pores) comes at the expense of a lower thermal conductivity. A CFC with high thermal conductivity cannot have the porosity needed for good neutron swelling resistance, and generally materials are optimized for one property or the other.

As porous carbon materials experience neutron damage to the graphite planes or carbon fibers, trapping sites are opened up, which can be saturated with hydrogenic fuel gas (deuterium and tritium). Retention of radioactive tritium is an area of concern for D-T fusion devices if the total inventory of tritium is substantially increased beyond what is necessary for fuel.

In evaluating candidate carbon materials for reactor wall structures, it is important to consider each material independently and completely, rather than relying on a combination of properties from different materials to satisfy a varied set of design requirements. Any given material may have some highly favorable properties at the expense of others. As mentioned above, N3M nuclear graphite (a Sombrero candidate material) exhibits lower than average neutron-induced swelling, but at the expense of lower thermal conductivity. In choosing 
candidate first-wall materials, it is critical to assess whether the design requirements are met by a single material.

\section{Sombrero Conditions}

The Sombrero target cavity chamber is an upright cylinder with conical ends (fig. 1). The size is such that a $6.5 \mathrm{~m}$ radius sphere could be inscribed in the chamber. The chamber wall is to be constructed of 12 modules, each covering $30^{\circ}$ of the horizontal plane and extending over the full height of the chamber ( $\sim 20$ meters). 60 beam ports are arranged symmetrically around the chamber, located along the interfaces between the 12 wall modules. The radial build of the wall consists of six layers of graphite separated by five layers of flowing granular $\mathrm{Li}_{2} \mathrm{O}$ and $\mathrm{He}$ gas, totaling $1 \mathrm{~m}$ thick at the midplane. The first-wall is a $1 \mathrm{~cm}$ thick layer of carbon-fiber composite, and the layer-averaged fraction of carbon increases as one goes outward through the layers. During operation, a strong temperature gradient exists from the inside to the outside of the chamber.

The Sombrero models ${ }^{(1)}$ derive a steady state and peak wall temperature using thermal conductivity of $140 \mathrm{~W} / \mathrm{m} \cdot \mathrm{K}$, degrading to $70 \mathrm{~W} / \mathrm{m} \cdot \mathrm{K}$ after irradiation. Peak heat flux on the wall is $1380 \mathrm{MW} / \mathrm{m}^{2}$. Using these figures, the peak wall temperature was calculated to be $2155^{\circ} \mathrm{C}$, decaying to a steady-state temperature of $1485^{\circ} \mathrm{C}$ in several $\mathrm{ms}$. The repetition rate is $6.7 \mathrm{~Hz}$, or $150 \mathrm{~ms}$ between shots. Neutron damage to the wall was estimated at $\sim 15$ dpa per full-poweryear (FPY). Using the Sombrero CFC lifetime estimate of $75 \mathrm{dpa}$, this would lead to a wall lifetime of $\sim 5$ FPY. 


\section{Thermal Conductivity}

CFC's have been demonstrated to have thermal conductivity $(\lambda)$ of up to several hundred $\mathrm{W} / \mathrm{m} \cdot \mathrm{K}$ at room temperature, before neutron irradiation ${ }^{(6)}$. Without irradiation, $\lambda$ degrades sharply with increasing temperature. This effect depends on the particular CFC, and generally the composites that have the highest room-temperature $\lambda$ experience the sharpest reduction at high temperatures. For example, as shown in figure 2, a composite with room-temperature $\lambda \sim 700 \mathrm{~W} / \mathrm{m} \cdot \mathrm{K}$ is reduced to $\lambda \sim 220 \mathrm{~W} / \mathrm{m} \cdot \mathrm{K}$ at $\mathrm{T}=1500^{\circ} \mathrm{C}$, for a reduction of $69 \%$. On the other hand, a composite with room-temperature $\lambda \sim 400 \mathrm{~W} / \mathrm{m} \cdot \mathrm{K}$ is reduced to $\lambda \sim 175 \mathrm{~W} / \mathrm{m} \cdot \mathrm{K}$ at $\mathrm{T}=1500^{\circ} \mathrm{C}$, for a reduction of $56 \%{ }^{(6)}$. The same study from which this data is taken includes an isotropic graphite (H451) sample which experienced a reduction from $\lambda \sim 120 \mathrm{~W} / \mathrm{m} \cdot \mathrm{K}$ at room temperature to $\lambda \sim 40 \mathrm{~W} / \mathrm{m} \cdot \mathrm{K}$ at $1200^{\circ} \mathrm{C}(67 \%$ reduction). Other studies have observed similar trends, though often with lower initial and final $\lambda$ values $^{(7)}$.

The pre-irradiation behavior of CFC's with the highest room-temperature thermal conductivity can be summarized by stating that temperature increases from room-temperature to $1500^{\circ} \mathrm{C}$ reduce $\lambda$ from $\sim 400-700 \mathrm{~W} / \mathrm{m} \cdot \mathrm{K}$ to $\sim 160-220 \mathrm{~W} / \mathrm{m} \cdot \mathrm{K}$. Although the relative temperature reduction and the final $\lambda$ value are dependent on the particular composite, the differences between the different composites are suppressed at high temperatures.

As mentioned above, neutron irradiation acts to degrade thermal conductivity, through a combination of temperature and radiation effects. At low temperatures, neutron irradiation causes a straightforward degradation of thermal conductivity (due to the low mobility of the interstitials and vacancies). Three studies are of particular interest ${ }^{(3,6,8)}$, wherein several different CFC samples were irradiated at $150^{\circ} \mathrm{C}, 400^{\circ} \mathrm{C}$ and $600^{\circ} \mathrm{C}$ up to damage levels of $17 \mathrm{dpa}$. The results for all materials tested were very similar, with saturation of the radiation-induced $\lambda$ 
degradation occurring by $\sim 0.1 \mathrm{dpa}$ at the lowest temperatures, and at $\sim 10 \mathrm{dpa}$ at the higher temperatures. Post-irradiation $\lambda$ values were $\sim 10 \%$ of the initial values in the lowest-temperature study, $20-30 \%$ of initial $\lambda$ in the higher temperature studies. Figure 3 shows representative behavior of $\lambda$ at $\mathrm{T}_{\text {irr }}=150^{\circ} \mathrm{C}$.

At higher temperatures $\left(\geq 1000^{\circ} \mathrm{C}\right)$, thermal annealing of radiation-induced lattice defects acts to increase the thermal conductivity, partially restoring the material to up to $80 \%$ of the unirradiated high-temperature value. Studies in which the neutron irradiation temperature is $400-600^{\circ} \mathrm{C}$ and the sample was subsequently heated above $1000^{\circ} \mathrm{C}$ demonstrate an initial reduction in $\lambda$ by $70-80 \%$ before the effect saturates, and a subsequent recovery of $\lambda$ to values very near the unirradiated high-temperature levels at temperatures above $1000^{\circ} \mathrm{C}^{(7,8,9)}$ (fig. 4). The temperature at which the materials are irradiated $\left(T_{\text {irr }}\right)$ is also seen to affect the material properties of CFC's. It has been shown that the relative degradation of $\lambda$ decreases with increasing $\mathrm{T}_{\text {irr }}{ }^{(10)}$. Again, the composites with highest initial $\lambda$ are still the highest after irradiation and heating, though the difference between different materials is suppressed at high temperatures.

Although the exact interplay between the effects of heat load and neutron irradiation is somewhat complex, the trend at high temperature is fairly simple. Despite the fact that neutron irradiation and high heat loads both independently degrade thermal conductivity in CFC's, the $\lambda$ values for all samples in this study (CFC's and graphites) seem to converge at high temperatures $\left(\geq 1000^{\circ} \mathrm{C}\right)$. Thermal conductivity for all irradiated CFC and graphite samples measured at $1500^{\circ} \mathrm{C}$ falls between 40 and $60 \mathrm{~W} / \mathrm{m} \cdot \mathrm{K}$, regardless of neutron exposure levels (tested from 0.01 dpa up to saturation of $\lambda$ degradation effects at $\sim 1 \mathrm{dpa}$ ) or initial thermal conductivity. 
It is important to note that the available data on high-temperature radiation-induced thermal conductivity degradation is somewhat scarce, and covers only a limited set of materials and conditions. In particular, radiation-damage data for the high-quality CFC's described in reference 6 and figure 2 is presently unavailable. The consistency of the graphite results can provide clues toward predicting CFC behavior under Sombrero-like conditions, but it should be noted that until the CFC data is available, this is only a best-guess.

With this in mind, it is interesting to look at the effect of thermal conductivity assumptions on temperature rise of the first wall. The Sombrero report models wall temperatures of $1758 \mathrm{~K}$ (steady-state) and $2428 \mathrm{~K}$ (peak), or $\Delta \mathrm{T}=670^{\circ}$. This level of temperature rise, based on the reported thermal flux to the wall, is consistent with $\lambda \approx 120 \mathrm{~W} / \mathrm{m} \cdot \mathrm{K}$; clearly a preirradiation/low-temperature level. (This value was derived independently from the Sombrero study, using the proposed chamber geometry. This is to be compared with the stated preirradiation $\lambda$ of $140 \mathrm{~W} / \mathrm{m} \cdot \mathrm{K}$, with the difference attributable to details in the thermal-transport models.) Modifying this calculation with a post-irradiation $\lambda$ of $50 \mathrm{~W} / \mathrm{m} \cdot \mathrm{K}$, a value consistent with the data mentioned above, $\Delta \mathrm{T}$ becomes $1038^{\circ}\left(\Delta T \propto \sqrt{\frac{1}{\lambda}}\right)$. This brings the peak temperature to $2796 \mathrm{~K}$, assuming the increased temperature rise does not cause additional "ratcheting up" of the steady-state temperature.

\section{Wall Erosion}

At first glance, the sensitivity of $\Delta \mathrm{T}$ to thermal conductivity may not seem particularly significant, but it is worth noting the effect on the carbon vapor pressure, which is highly temperature dependent, and the resulting effect on wall erosion rates due to the incident heat flux to the first wall. Using the carbon vapor pressure formula $P=P_{0} \cdot \exp \left\{\left(-9.2 \cdot 10^{4}\right) /[T(K)]\right\}$ with 
$P_{0}=4.156 \cdot 10^{12}$ torr, one finds that $\mathrm{P}(2428 \mathrm{~K})=1.4 \cdot 10^{-4}$ torr, and $\mathrm{P}(2796 \mathrm{~K})=2.1 \cdot 10^{-2}$ torr (these correspond to the peak wall temperatures predicted in the Sombrero report and using $\lambda=50$ $\mathrm{W} / \mathrm{m} \cdot \mathrm{K}$, respectively). The factor of 1.55 increase in $\Delta \mathrm{T}$ induced by neutron irradiation (through thermal-conductivity degradation) translates into a 150 -fold increase in carbon vapor pressure at the wall.

One can then estimate wall erosion rates using a simple thermal-evaporation approach. Here the assumption is made that the vaporized carbon is not redeposited in the same location as the evaporation. There are two likely possibilities for the destination of carbon vapor in the Sombrero test chamber. First, Sombrero must have an extremely efficient chamber-clearing system, which will quickly remove the carbon vapor from the system along with the fuel pellet debris. In the event that the vapor is not immediately swept out, one can assume that it will recondense in a colder region of the chamber. The midplane of the Sombrero chamber is $6.5 \mathrm{~m}$ from the center, while the top and bottom are $9 \mathrm{~m}$ from the center. This translates to $\sim 50 \%$ lower heat-flux at the top and bottom than at the midplane. With this in mind, one can consider the following erosion calculations to be relevant at the midplane, with any corresponding recondensation occurring off-midplane.

Using a kinetic-gas treatment, one can derive an estimate for first-wall erosion due just to thermal evaporation. The evaporated carbon will fill a volume that is limited by diffusion through the xenon background gas, with the diffusion distance calculated as follows: Taking the xenon interparticle spacing to be $\sim 0.8 \mu \mathrm{m}$ (at 0.5 torr), the xenon radius as $150 \mathrm{pm}$, and the carbon thermal velocity as $\sim 2.3 \mathrm{~km} / \mathrm{s}$, one arrives at a diffusion rate of carbon through xenon of $\mathrm{D} \sim 60 \mathrm{~m}^{2} / \mathrm{s}$. Translating this into linear diffusion from the wall during a $0.5 \mathrm{~ms}$ high- 
temperature period, this yields a diffusion distance of $\sim 5 \mathrm{~cm}$ (unlike the vapor pressure, this figure has only a weak dependence on carbon temperature over the range considered).

With a $5 \mathrm{~cm}$ layer containing evaporated carbon at the temperature-dependent partial pressure calculated above, one can estimate the mass of evaporated carbon per shot, and thus the erosion depth per shot. For $\mathrm{T}_{\text {peak }}=2428 \mathrm{~K}$, the evaporated mass comes to $0.58 \mu \mathrm{g}$ per shot, for an erosion rate of $0.07 \mathrm{~mm} / \mathrm{yr}$. For $\mathrm{T}_{\text {peak }}=2796 \mathrm{~K}$, on the other hand, the evaporated mass comes to $73 \mu \mathrm{g}$ per shot, for an erosion rate of $8.8 \mathrm{~mm} / \mathrm{yr}$. At this rate, the $1 \mathrm{~cm}$ first wall would be completely eroded in 14 months. It should be noted that this estimate includes only vaporization, and no ablation of larger particles, which would further accelerate erosion.

Again, radiation-damage data for high-quality CFC's is presently unavailable, and the preceding evaporation estimate is merely a "back-of-the-envelope" calculation based on a bestguess of thermal conductivity. Until data for specific candidate materials is available for Sombrero-like conditions, it is nearly impossible to make accurate estimates of erosion rates in the Sombrero environment. The above evaporation/erosion calculation is meant to illustrate the extreme sensitivity of wall erosion rates to peak wall temperature, and thus to the thermal conductivity of the first-wall material.

Experimental data on carbon erosion from fast heat pulses with parameters exactly matched to the Sombrero conditions is also presently unavailable. Although there have been several studies relevant to disruption events in magnetic-fusion devices, none cover the combination of high power $\left(\sim 1 \mathrm{GW} / \mathrm{m}^{2}\right)$ and low energy $\left(0.1 \mathrm{MJ} / \mathrm{m}^{2}\right)$ predicted for Sombrerotype heat pulses. This said, data does exist for graphites and CFC's under short pulse, high heat flux conditions at 6-30 GW/m $/ \mathrm{m}^{2}$ and 2-10 MJ $/ \mathrm{m}^{2}$, taken at a Nd:YAG laser facility ${ }^{11}$. The data shows a threshold energy for erosion, above which there is a linear dependence of erosion depth 
on absorbed energy density, or $\delta=\mathrm{K} \cdot\left(\mathrm{E}-\mathrm{E}_{0}\right)$. The linear coefficient $\mathrm{K}$ ranges from 13-17 $\mu \mathrm{m} \cdot \mathrm{m}^{2} / \mathrm{MJ}$

The threshold $\mathrm{E}_{0}$ was observed to range between $0.5-2.0 \mathrm{MJ} / \mathrm{m}^{2}$, depending on the material. The data was found to agree fairly well with a finite-element model which solved a 1D heat equation using temperature-dependent material properties, surface evaporation, radiation, and surface recession. Because the data agrees with a straightforward evaporation/radiation model, one might assume that for samples initially at temperatures approaching the threshold for sublimation, $\mathrm{E}_{0}$ may be quite low, while the linear dependence on incident energy would remain.

Without actual experimental conditions that mimic the Sombrero environment, it is extremely difficult to form an erosion estimate from this data. For example, the samples in the above experiment were initially at room temperature and under vacuum. The Sombrero-relevant scenario would include target carbon that is 'pre-warmed' to a temperature close to that of sublimation, but in a background fill of 0.5 torr of xenon. The rate of erosion would then presumably follow similar behavior to that mentioned above, though with a reduced $E_{0}$. With pulses coming at 6.7 per second, the erosion rate is critically dependent on $E_{0}$, which is itself strongly dependent on the high-temperature, neutron-damaged thermal conductivity of the carbon wall. The compounding uncertainties in these quantities makes any extrapolation from the present experimental data unreliable.

This again demonstrates the striking dependence of erosion rates to material temperature and thermal conductivity. In the past, it has been assumed that the per-pulse carbon evaporation would be low enough as to be neglected, but this appears to be a dangerous assumption.

In conclusion, we find that simple calculations based on post-irradiation thermal conductivity values can yield erosion-rates of $8.8 \mathrm{~mm} / \mathrm{yr}$ or more, corresponding to first-wall 
lifetimes of $\sim 14$ months or less. Experimental data corresponding to the exact combination of Sombrero conditions is presently unavailable, and we summarize this section by simply stating that without such data, erosion rates are very difficult to predict, can vary widely with different materials (CFC's and graphites), are critically temperature dependent, and could potentially limit the first-wall lifetime.

\section{Dimensional Changes}

Another major effect of neutron irradiation on graphite and CFC's is bulk contraction and expansion. Individual fiber and fiber-lattice damage induced by neutron irradiation can cause shrinkage and swelling of materials of $10-20 \%$ before the damage causes excessive pores and cracks and the material completely disintegrates.

Because of the anisotropic structure of CFC materials, dimensional changes are demonstrated to be very different than in graphite, and highly dependent on axis direction. One model of the individual carbon-fiber is that it is composed of a series of coaxial sheaths around a straight core. At low neutron fluences, radiation-induced shrinkage is primarily within the graphitic planes (axial and azimuthal directions on the fiber), while swelling in the "radial" direction can be initially accommodated by either interplanar voids and pores or by the network of cracks between the fibers and the matrix. Thus the initial effect of neutron radiation is volumetric shrinkage, primarily in the axial direction of the fibers ${ }^{(12)}$. Because the shrinkage is non-isotropic, internal stresses can also form, and "bowing" of samples has been observed at low fluence levels $(<2 \mathrm{dpa})^{(13)}$. It should be noted that the "core-sheath" model is one of several models for the microstructure of carbon fibers, and that different CFC's have different matrix structures. In all cases, though, CFC's with any microstructure exhibit the general properties associated with anisotropy. 
This has been demonstrated in several experiments ${ }^{(3,7,10,12,13)}$, wherein samples were irradiated at levels up to $4.7 \mathrm{dpa}$, and dimensional shrinkages were observed up to $4 \%$ in the "preferred direction" and $1.8 \%$ in the other directions. It was also demonstrated that changes along the fiber axis are higher for CFC's than for graphites by up to an order of magnitude (typically $2-3 x$ ) due to the anisotropy. Figure 5 shows typical behavior of CFC length shrinkage induced by neutron irradiation $<5 \mathrm{dpa}, \mathrm{T}_{\mathrm{irr}}=600^{\circ} \mathrm{C}$.

At higher fluence rates ( $>5 \mathrm{dpa}), \mathrm{CFC}$ 's undergo a "turnaround", when the rate of shrinkage slows and stops, and the material begins to re-expand. This has been explained as an increase in the porosity of the material parallel to the graphitic planes which becomes more pronounced with increasing neutron damage. Not only does the material continue to swell with increasing neutron fluence (eventually swelling past the initial unirradiated volume), but the rate of swelling also increases. N3M graphite, a material optimized to minimize neutron-induced swelling, has been modeled to reach volume-turnaround at $\sim 45 \mathrm{dpa}$. CFC's optimized for other properties (i.e. thermal conductivity or thermal shock resistance) do not have the internal porosity of N3M, and thus experience volume-turnaround at lower fluence levels.

This effect is also clearly dependent on $T_{\text {irr }}$, with higher $T_{\text {irr }}$ corresponding to lower neutron fluence required to reach volume-turnaround and also $\Delta \mathrm{V}$ crossing through zero. This effect has been attributed to the thermally induced closure of interplanar voids within the matrix, which reduces their ability to accommodate expansion along the fiber axis ${ }^{(5)}$. The point at which $\Delta \mathrm{V}$ crosses through zero is often used as an estimate for the material lifetime, since at this point pores and cracks in the material due to differential strain within the material cause total disintegration ${ }^{(2)}$. It should be noted that this criterion has traditionally been used mainly out of convenience, since it is known that beyond this point, materials expand without limit. For some 
materials, though, other factors may limit the practical lifetime before the zero-crossthrough point. For example, in structures with thermal or mechanical stresses the practical limit will be at a lower fluence as mechanical properties degrade more rapidly.

Experiments documented in references 5 and 14 demonstrate this dependence of $\Delta \mathrm{V}$ on $\mathrm{T}_{\text {irr. }}$. In one study, the $\Delta \mathrm{V}_{\max }$ observed at $\mathrm{T}_{\text {irr }}=875^{\circ} \mathrm{C}$ is approximately $60 \%$ of that observed at $\mathrm{T}_{\text {irr }}=600^{\circ} \mathrm{C}$, and occurred at $25 \%$ lower fluence ( $15 \mathrm{dpa}, 20 \mathrm{dpa}$ respectively). The point at which $\Delta \mathrm{V}$ crossed through zero was extrapolated to $22 \mathrm{dpa}$ for $\mathrm{T}_{\text {irr }}=875^{\circ} \mathrm{C}$ and $33 \mathrm{dpa}$ for $\mathrm{T}_{\text {irr }}=600^{\circ} \mathrm{C}$. Other nuclear graphites referenced (5) have shorter lifetimes; $15-17$ dpa at $875^{\circ} \mathrm{C}$.

In reference 13 , several CFC's were irradiated at $1500^{\circ} \mathrm{C}$ to levels up to $5.2 \mathrm{dpa}$, and the resultant dimensional changes reported. Dimensional changes in the direction perpendicular to the fiber direction are presented in figure 6. Perpendicular data is presented because this is assumed to be the most relevant to the Sombrero reactor geometry; it is assumed that thermal conductivity is to be maximized (parallel to fibers) normal to the chamber walls, which leaves the wall module length subject to dimensional changes perpendicular to the fibers. In addition to the plotted expansion (perpendicular to the fiber direction), there was a corresponding shrinkage parallel to the fiber direction(s), as described above, indicating that this perpendicular expansion is occurring before volume-turnaround. All CFC samples experience perpendicular expansion of $\sim 20 \%$ at damage levels $\leq 5$ dpa.

As with thermal conductivity effects, radiation-induced swelling is highly dependent on $\mathrm{T}_{\text {irr }}$. Samples of A05 irradiated to $1.6 \mathrm{dpa}$ at $\mathrm{T}_{\text {irr }}=600^{\circ} \mathrm{C}$ demonstrated $1 \%$ perpendicular shrinkage, but at $\mathrm{T}_{\text {irr }}=1500^{\circ} \mathrm{C}$ demonstrated $10 \%$ swelling $^{(14)}$.

It should be noted that the 3-D CFC's in the above data set (SEPCARB-A and NOVOLTEX) are not truly balanced materials. Both consist of 2-D layers in one plane with 
subsequent fiber stitching in the third direction, and are sometimes referred to as 'pseudo 3-D'. It is possible that a truly balanced 3-D CFC (such as FMI-222, for which unirradiated thermal conductivity data is shown in figure 2) would undergo significantly less swelling prior to volume-turnaround.

Data in reference 8 is consistent with the previous plot, with A05 exhibiting $20 \%$ swelling at 5 dpa neutron damage. Reference 9 documents the volume-turnaround point of $\mathrm{H}-$ 451 graphite under two different conditions, further demonstrating the strong dependence on $\mathrm{T}_{\text {irr. }}$. The samples exhibited turnaround for $\mathrm{T}_{\mathrm{irr}}=620^{\circ} \mathrm{C}, 900^{\circ} \mathrm{C}$ at $16 \mathrm{dpa}, 10 \mathrm{dpa}$, respectively, and $\Delta \mathrm{V}$ was extrapolated to cross through zero at $29 \mathrm{dpa}, 17 \mathrm{dpa}$, respectively.

Despite some variation in swelling behavior from sample to sample in the tested CFC's, it is clear that expansion perpendicular to the fibers can be quite significant $(\sim 20 \%)$ even at neutron levels predicted to be reached in several months in the Sombrero reactor. This has a direct impact on the usable lifetime of the CFC wall materials, which will be further shortened by the high temperature at which the walls are irradiated.

The mechanical stress induced in the structure due to the shrinkage and the high elastic modulus of the CFC are an additional concern. The Sombrero design calls for the target chamber to be comprised of monolithic CFC modules, $20 \mathrm{~m}$ high, which will be subject to neutron damage levels which vary from the front surface to the back. Swelling of the innermost layers of up to $20 \%$ in the direction perpendicular to the carbon fibers would make the engineering of such modules technically very difficult. With this in mind, it is critically important to investigate the expansion properties of truly balanced 3-D CFC materials, which could potentially undergo significantly less pre-turnaround expansion. Usability of CFC's for which swelling data is presently available is a major concern. 


\section{Tritium Retention}

Another effect of neutron damage in carbon materials is that of increased tritium retention levels. This is a concern in any tritium-based fusion device, since trapping of tritium in the facing components increases the total inventory of radioactive material while not contributing to the fuel inventory.

Neutron radiation effects on tritium retention in graphite have been simulated in the past using carbon-ion irradiation, though recent work (not available at the time of the Sombrero report) has been published using actual neutron irradiation, with both graphite and CFC's ${ }^{(15,16)}$. Figure 7 shows the level of saturated tritium retention (atomic ppm) as a function of neutron damage for three CFC's and four graphites. Despite the wide variations in retention levels, one can observe a general trend towards increased retention at higher radiation damage levels, with possibly some saturation at high damage levels. It is also clear that, in general, CFC retention is lower than that of graphite.

Of particular note is N3M graphite, previously mentioned as the candidate Sombrero wall material. This graphite has one of the highest tritium retention levels of the materials shown. Assuming that half of the retention is deuterium and half is tritium, a level of $1000 \mathrm{appm}$ in N3M graphite would translate to tritium retention at 125 grams per metric ton. Even at more favorable levels of 100 appm, retention would be 12.5 grams per metric ton.

The neutron damage in the graphite wall has an e-folding length of about $20 \mathrm{~cm}$, and the percentage of carbon increases through the thickness of the wall. Using the amount of carbon in each layer, the anticipated neutron damage as a function of distance form the first wall, and the temperature of each wall section, we estimate the tritium retention to be between 0.5 and $5.0 \mathrm{~kg}$ by the time the front surface reaches $10 \mathrm{dpa}$ ( $\sim 8$ months of Sombrero full-power operation), with 
the majority of tritium located in the second and third graphite layers. Well before the end of life, these sections will have been exposed to sufficient neutron damage to saturate the production of tritium traps. These sections will also have temperatures sufficient to allow tritium diffusion into the graphite grains, but not so high as to prevent the decoration of the high energy traps.

Another consideration is the transport of tritium in the irradiated walls. Tritium and deuterium that remains unburned from a pulse in the Sombrero chamber will strike the graphite wall with an energy of a few eV. With the inner graphite wall section at temperatures near 2200 $\mathrm{K}$, the hydrogen isotopes embedded in the wall will rapidly be released in the atomic form. Much of this atomic hydrogen will re-enter the wall and rapidly migrate through the porous graphite. Deuterium and tritium emerging from the rear of the first wall section will recombine into molecules and join the tritium being released from the lithium oxide in the first coolant section. All of this gas will then serve as the source for permeation through the next wall layer, and the process will be continued through all of the layers. The fact that the outer wall sections are not as hot as the inner sections does not mean that permeation does not occur. Kiyoshi et $a l{ }^{(17)}$ has shown the permeation rate of tritium through graphite to actually increase as the temperature was decreased. Both permeation through the outer wall section and the kg-level bulk tritium retention may be serious safety issues for Sombrero.

It may be possible to develop methods by which coatings such as $\mathrm{B}_{4} \mathrm{C}$ or $\mathrm{SiC}$ could be used to coat the inner wall of the chamber to reduce the neutron penetration, though studies are needed to assess the feasibility of such coatings when applied to material undergoing neutroninduced swelling at the level predicted in Sombrero. 


\section{Summary}

In choosing candidate first-wall materials, it is critical to assess whether the design requirements are met by a single material, and care must be taken not to rely on a combination of properties from different materials to satisfy a varied set of design requirements. Any given material may have some highly favorable properties at the expense of others. For example, N3M nuclear graphite, a Sombrero candidate wall material, is optimized for low radiation swelling, but at the expense of lower thermal conductivity and relatively high tritium retention.

Based on the available literature concerning neutron radiation effects on carbon materials, the Sombrero reactor design raises three primary issues of concern:

1. The assumed thermal conductivity values of $140 \mathrm{~W} / \mathrm{m} \cdot \mathrm{K}$ (before irradiation) and 70 $\mathrm{W} / \mathrm{m} \cdot \mathrm{K}$ (after irradiation) seem optimistic, and modeling of the wall temperature-rise caused by the pulsed heat flux based on unirradiated thermal conductivity values may lead to inaccurate assumptions about the carbon sublimation rates, which are highly temperature dependent. Recalculation of the temperature rise based on a post-irradiation best-guess conductivity value of $50 \mathrm{~W} / \mathrm{m} \cdot \mathrm{K}$ leads to a 150 -fold increase in wall carbon vapor pressure. Erosion estimates are dramatically dependent on the assumed thermal conductivity of the wall material, as demonstrated by an example calculation based on thermal conductivity of $50 \mathrm{~W} / \mathrm{m} \cdot \mathrm{K}$, which predicts complete erosion of the $1 \mathrm{~cm}$ first wall layer within 14 months or less. A material may be developed with a high-temperature thermal conductivity value higher than $50 \mathrm{~W} / \mathrm{m} \cdot \mathrm{K}$, but the cost to other material properties must be critically weighed. In any case, the sensitivity of erosion rates to thermal conductivity and wall temperature warrants further investigation into the erosion issue. 
2. Radiation-induced swelling poses another possible problem with the use of carbon materials for the chamber walls. Data from 2-D and 'pseudo 3-D' CFC's consistently demonstrate swelling of $\sim 20 \%$ with neutron damage of $5 \mathrm{dpa}$, a level expected to be reached within several months in Sombrero. This level of swelling, particularly in view of the front-toback variation, could make the engineering of the CFC target-chamber modules technically difficult. High temperature neutron irradiation data is presently only available for 2-D and "pseudo 3-D" CFC's, and it is possible that true 3-D or 4-D advanced CFC's may demonstrate improved swelling characteristics. Data for these advanced weaves, perhaps from a nuclear fission reactor, is clearly needed to sufficiently address this issue.

The useful carbon-wall lifetime of $75 \mathrm{dpa}$, presented in the Sombrero report using an extrapolated parameter-scaling model, is not supported by the experimental data on irradiation of CFC's. The high irradiation temperature in the reactor may limit CFC lifetimes to 15-30 dpa, or may be additionally limited by other effects.

3. Though there is quite a bit of variability in the tritium retention properties of different carbon materials, one might reasonably expect retention of 100-1000 appm tritium in carbon after neutron irradiation based on presently available data. This would lead to $\sim 0.5$ to $5 \mathrm{~kg}$ of tritium retained in the Sombrero chamber walls within 8 months of full-power operation. This may call into question safety-status assumptions based on low tritium inventory, and may lead to problems associated with the permeation of the tritium out of the walls into the surrounding area. The issue of tritium inventory is of course not limited to Sombrero, but is a concern for any tritium-based fusion reactor design.

These results indicate the urgency of high-temperature neutron-irradiation tests of fully symmetric 3-D CFC candidate materials. It is critical to determine the thermal conductivity, 
erosion, swelling, and tritium retention properties of such materials under neutron irradiation and high heat loads in order to support the plausibility of a carbon first-wall IFE reactor chamber such as proposed for Sombrero.

\section{Acknowledgements}

I would like to thank M. Ulrickson, R. Causey, R. Goldston, R. F. Mattas, L. L. Snead, and K. Wilson for many helpful technical discussions. 


\section{Figure Captions}

Figure 1. The Sombrero target chamber. (From ref. 1)

Figure 2. Thermal conductivity vs. temperature for several unirradiated CFC's (FMI-1D, MKC1PH, FMI-222) and graphites (H451, RGTi). (From ref. 6)

Figure 3. Normalized thermal conductivity vs. damage level at low $\left(150^{\circ} \mathrm{C}\right)$ temperature. (From ref. 6)

Figure 4. Thermal conductivity vs. temperature for two graphites (IG-110U, ETP-10) and one C-C composite (CX-2002U). Behavior of all samples converges at high temperature. (From ref. 7)

Figure 5. Length changes vs. neutron damage for two 3-D CFC's (FMI-222, FMI-223) which had been heat treated (pre-irradiation) to $2650{ }^{\circ} \mathrm{C}$ or $3100{ }^{\circ} \mathrm{C}$. $\mathrm{T}_{\text {irr }}$ for these samples was $600^{\circ} \mathrm{C}$. (From ref. 3)

Figure 6. Dimensional change vs. neutron damage for 2-D CFC's (A05, A21), and 3-D CFC's (SEPCARB-A, NOVOLTEX) irradiated at $1500^{\circ} \mathrm{C}$. (Data from ref. 14) 
Figure 7. Tritium retention vs. neutron damage for CFC's (FMI-222, MKC-1PH, A05), and graphites (N3M, S1611, CLS5890, Pfizer pyrolytic) irradiated at $200^{\circ} \mathrm{C}<\mathrm{T}_{\mathrm{irr}}<1400^{\circ} \mathrm{C}$. (Data from refs. 15,16) 


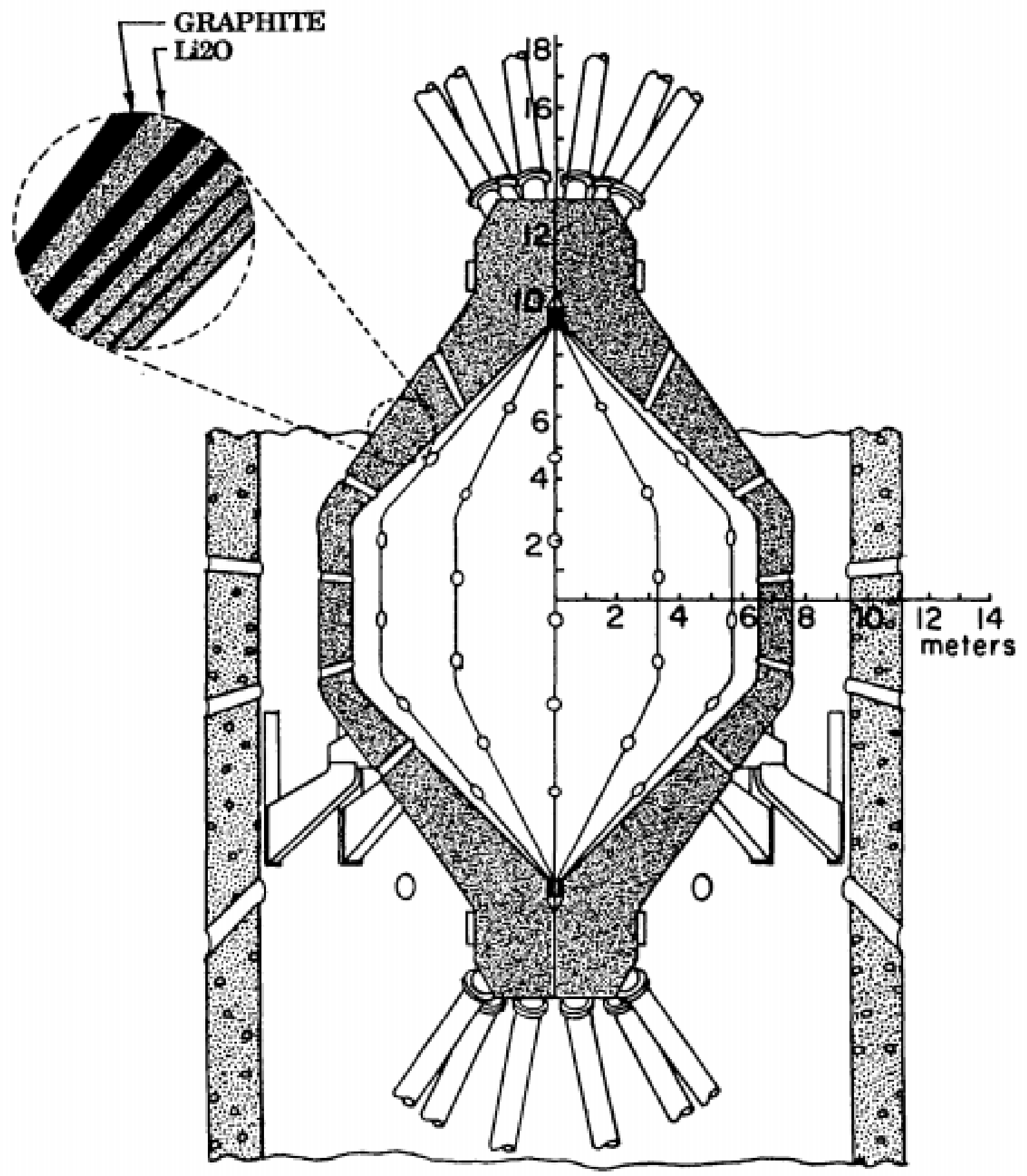

Figure 1 


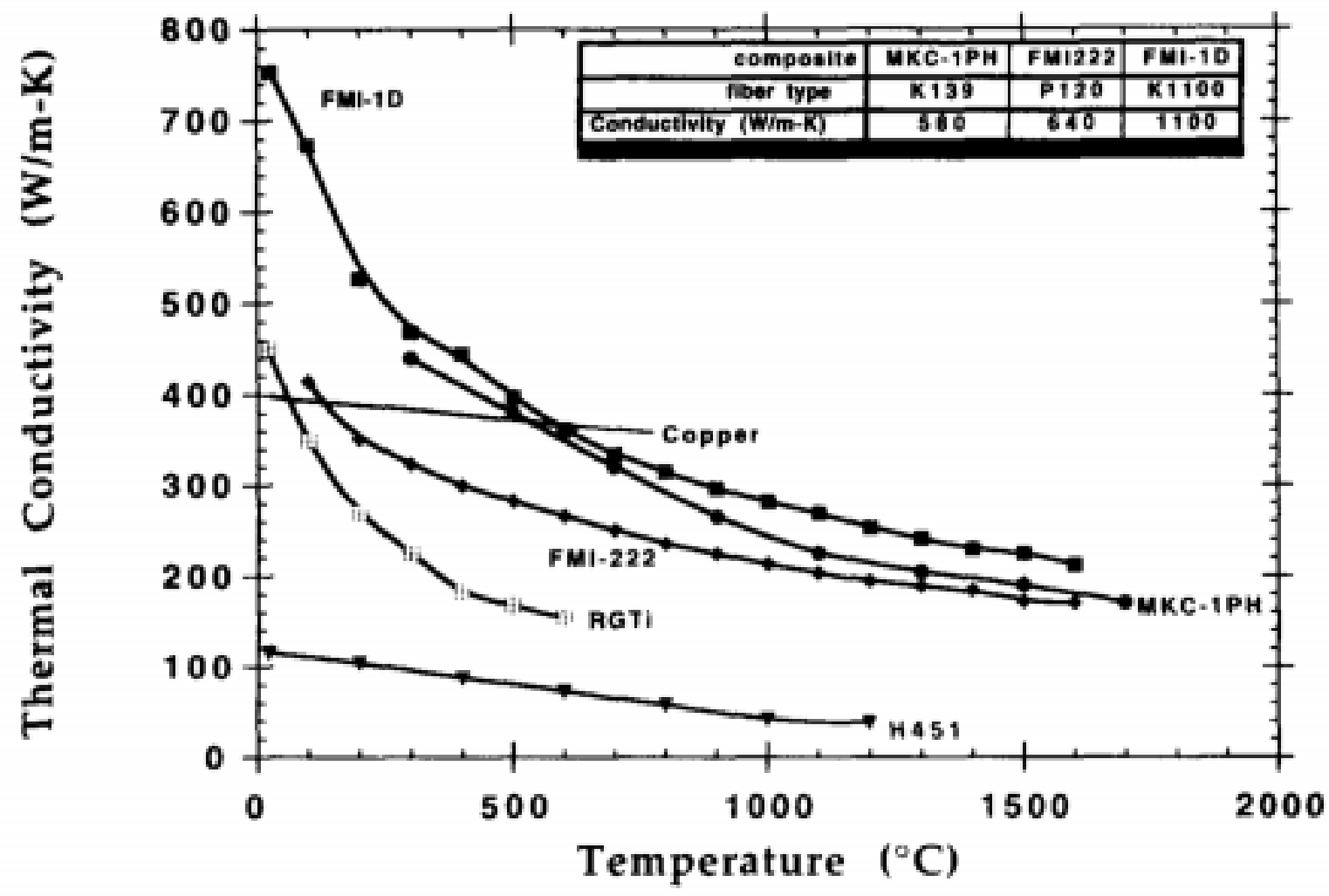

Figure 2 


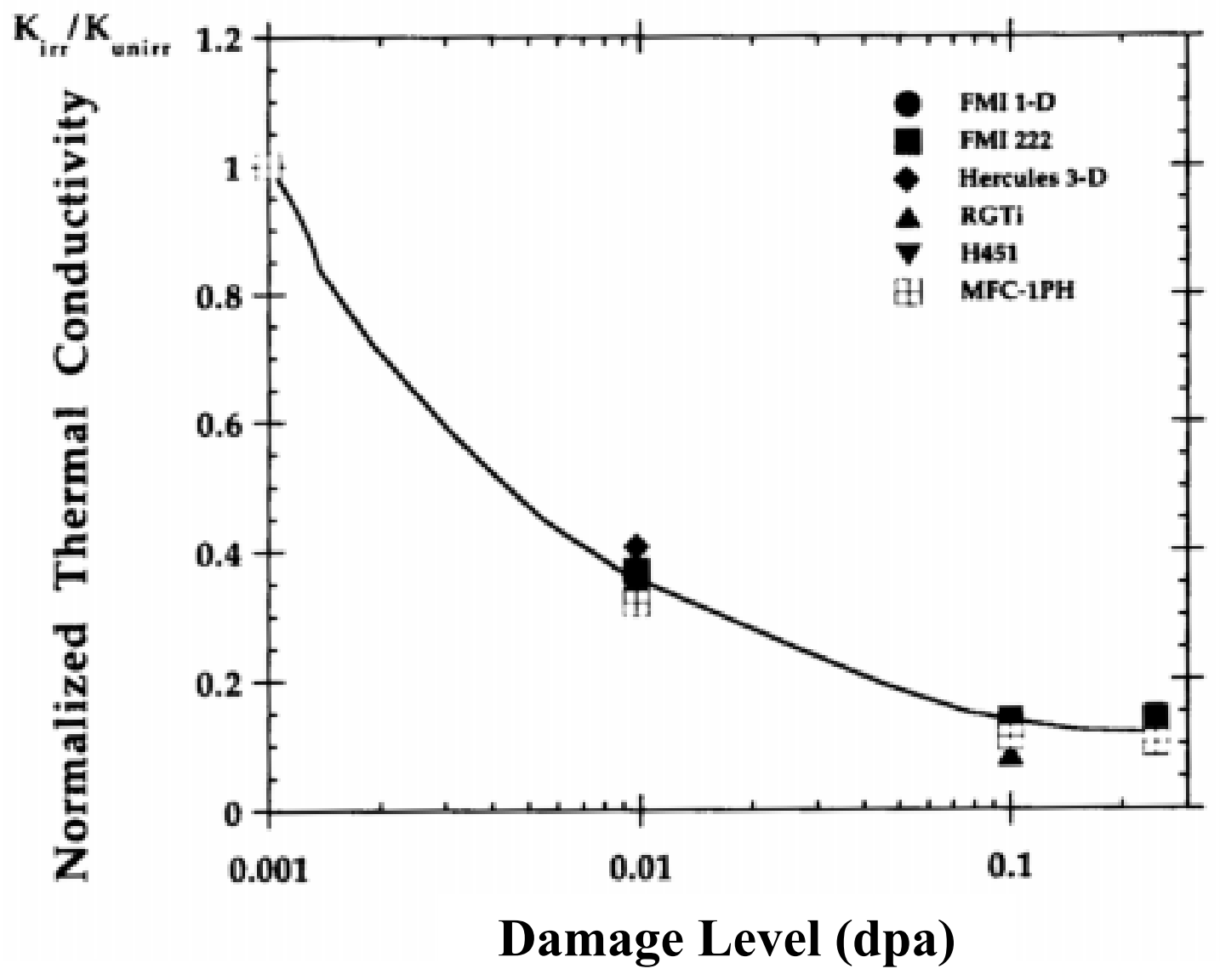

Figure 3 


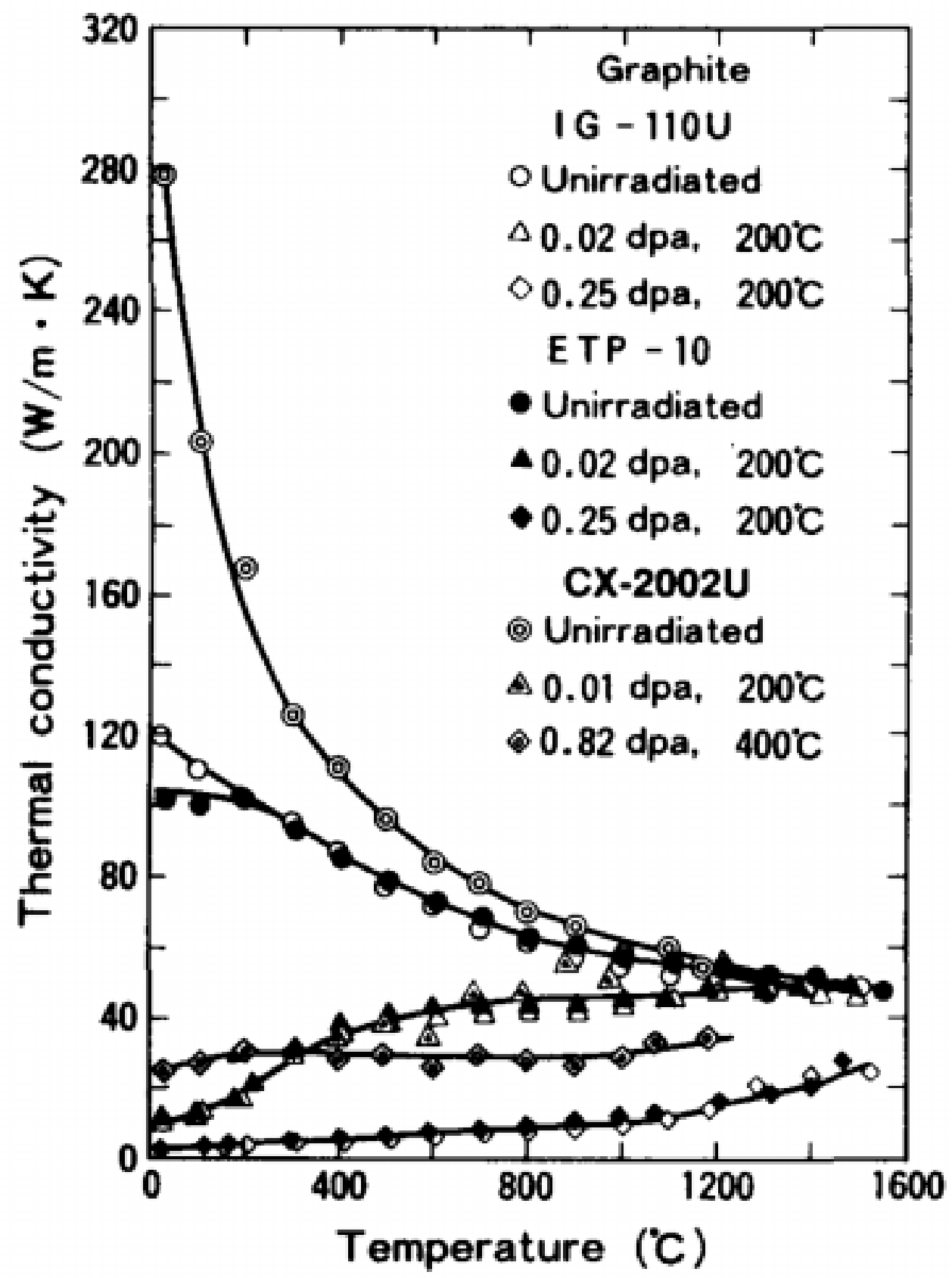

Figure 4 


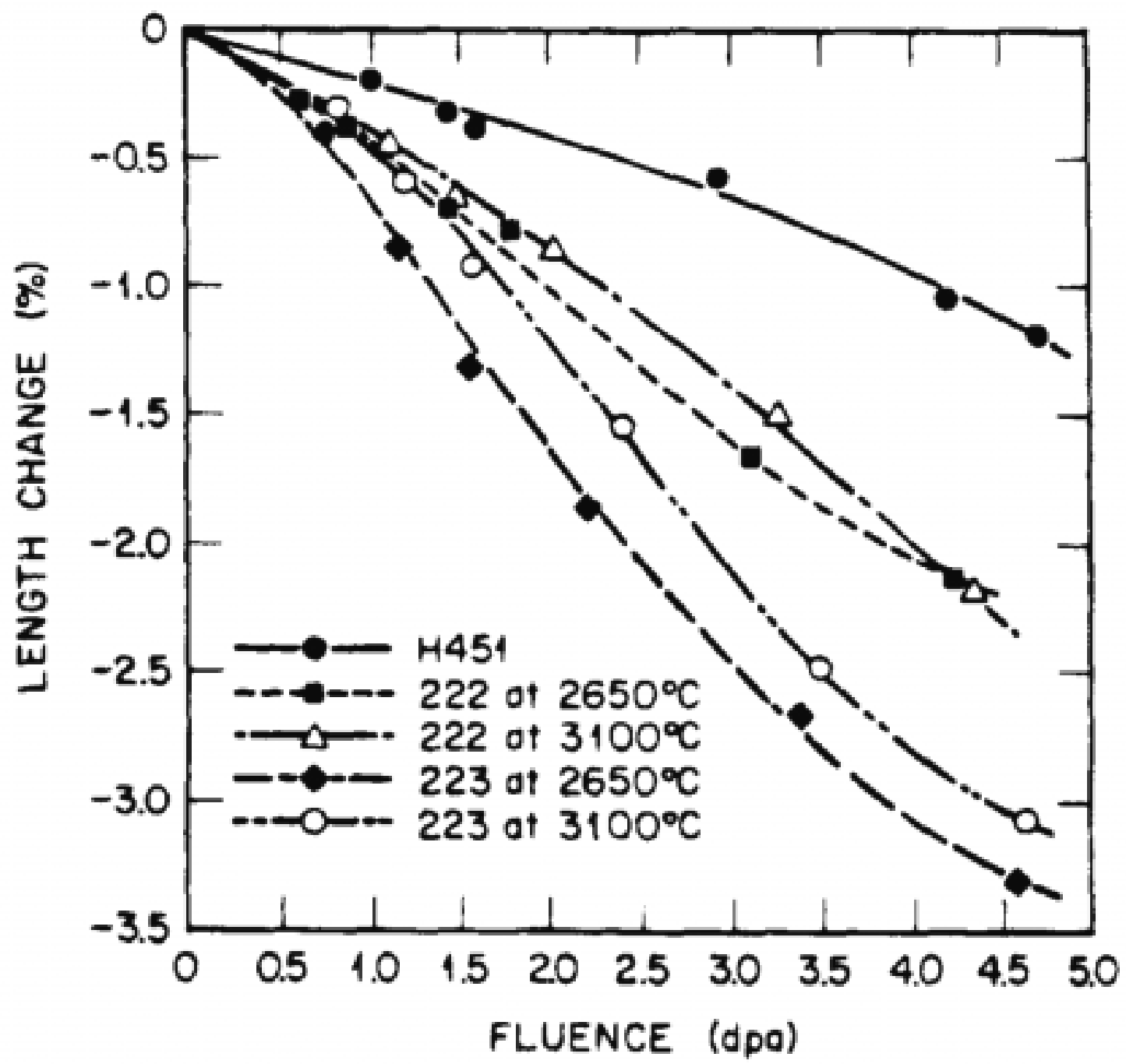

Figure 5 


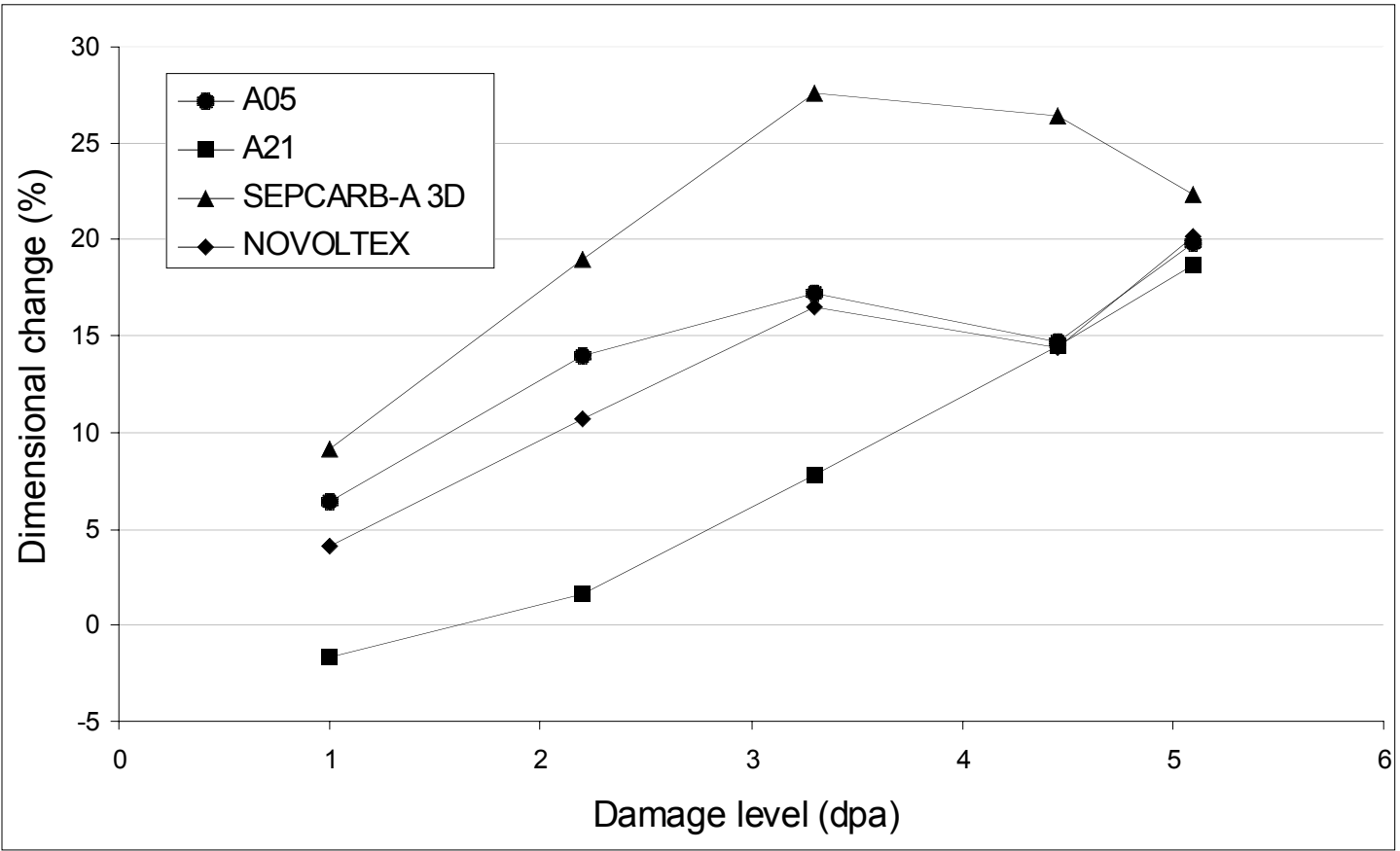

Figure 6 


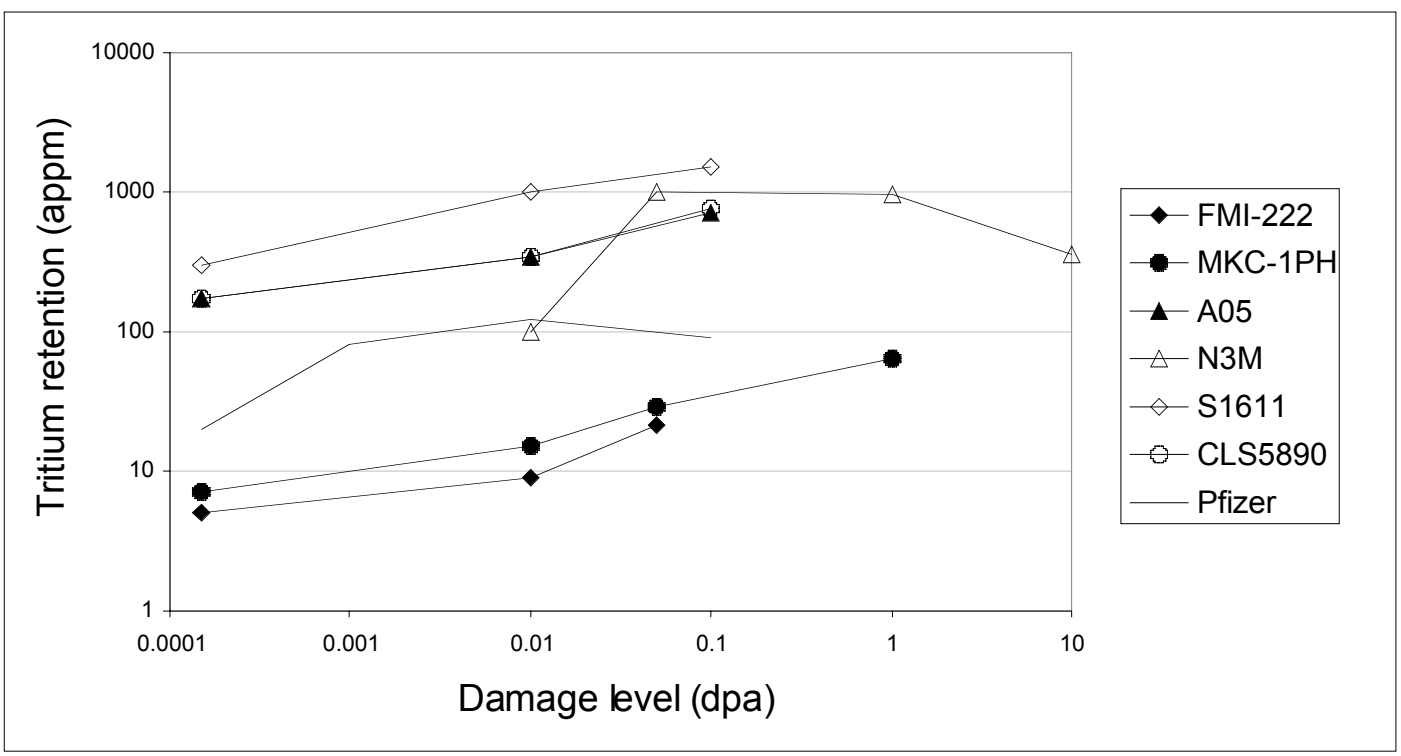

Figure 7 


\section{References}

${ }^{1}$ OSIRIS and SOMBRERO Inertial Fusion Power Plant Designs, W. J. Schafer Associates, WSJA-92-01, DOE/ER/54100-1 (1992).

${ }^{2}$ T.D. Burchell, Radiation Damage in Carbon Materials, in Physical Processes of the

Interaction of Fusion Plasmas with Solids, Academic Press, NY, pp. 341-384 (1995).

${ }^{3}$ T.D. Burchell, Physica Scripta T64 pp. 17-25 (1996).

${ }^{4}$ B.T. Kelley, Physics of Graphite, Applied Science Publishers, Englewood, NJ, 1981, pp. 386460.

${ }^{5}$ T.D. Burchell and W.P. Eatherly, J. Nucl. Mater. 179-181 pp. 205-208 (1991).

${ }^{6}$ L.L. Snead and T.D. Burchell, J. Nucl. Mater. 224 pp. 222-229 (1995).

${ }^{7}$ T. Maruyama and M. Harayama, J. Nucl. Mater. 195 pp. $44-50$ (1992).

${ }^{8}$ C.H. Wu, et al, J. Nucl. Mater. 212-215 pp. 416-420 (1994).

${ }^{9}$ R.F. Mattas, Fus. Tech. 15 pp. 637-642 (1989).

${ }^{10}$ J.P. Bonal and C.H. Wu, Physica Scripta T64 pp. 26-31 (1996).

${ }^{11}$ J.G. van der Laan, et al, J. Nucl. Mater. 196-198 pp. 612-617 (1992).

${ }^{12}$ T.D. Burchell, et al, J. Nucl. Mater. 191-194 pp. 295-299 (1992).

${ }^{13}$ M. Eto, et al, J. Nucl. Mater. 212-215 pp. 1223-1227 (1994).

${ }^{14}$ J.P. Bonal, et al, J. Nucl. Mater. 212-215 pp. 1218-1222 (1994).

${ }^{15}$ R. Causey, et al, Physica Scripta T64 pp. $32-35$ (1996).

${ }^{16}$ H. Kwast, et al, Physica Scripta T64 pp. 41-47 (1996).

${ }^{17}$ T. Kiyoshi, T. Namba and M. Yamawaki, J. Nucl. Mater. 155-157 pp. 230-233 (1988). 